\title{
PROJECTIVE NORMAL FLATNESS AND HILBERT FUNCTIONS
}

BY

\author{
U. ORBANZ AND L. ROBBIANO
}

\begin{abstract}
Projective normal flatness of a local ring $R$ along an ideal $I$ is defined to be the flatness of the morphism on the exceptional divisor induced by blowing up $R$ with center $I$. It is shown that most of the important properties of normal flatness have an analogue for projective normal flatness. In particular, we study the local Hilbert function in connection with projective normal flatness. If $R / I$ is regular and $R$ projectively normally flat along $I$, then we obtain the same inequality for the local Hilbert functions under blowing up as in the permissible case.
\end{abstract}

Introduction. The notion of normal flatness was introduced by Hironaka in his famous paper [9] in order to control the numerical data of a singularity under monoidal transformations. The assumption of normal flatness implies, in particular, that the corresponding monoidal transformation induces a flat morphism on the exceptional divisor. If the local ring at the singularity is $R$ and if the center of the monoidal transformation is defined by the ideal $I \subset R$, then the morphism on the exceptional divisor is (locally) given by $\operatorname{Proj}\left(G_{I}(R)\right) \rightarrow \operatorname{Spec}(R / I)$, where $G_{I}(R)$ denotes the associated graded ring of $R$ with respect to $I$. In this paper we study the flatness of this morphism, and the first observation is that it is equivalent to the flatness of $I^{n} / I^{n+1}$ over $R / I$ for large $n$ (Proposition (1.2)). Therefore, this algebraic condition is investigated, and it is given the name projective normal flatness (of $R$ along $I$ ). The present paper consists mainly in showing that projective normal flatness shares all of the important properties of normal flatness, although sometimes additional assumptions are necessary.

One way of characterizing normal flatness is by the so-called HironakaGrothendieck isomorphism. A systematic account of the results on this isomorphism is now available in [13]. The main difficulty in translating these methods to our situation consists in the fact that many of the classical proofs use induction on the degree in $G_{I}(R)$, whereas for projective normal flatness only large degrees are considered. The main tool to overcome this difficulty is Proposition (1.9).

In [6] the question was raised for a reasonable condition "between" equimultiplicity and normal flatness, and indeed our projective normal flatness is such a condition, for we have the implications normal flatness $\Rightarrow$ projective normal flatness $\Rightarrow$ equimultiplicity [7, Theorem 1]: As an application of the techniques of $\$ 1$ we can

Received by the editors September 22, 1982.

1980 Mathematics Subject Classification. Primary 14B25, 13H15; Secondary 13C15.

Key words and phrases. Normal flatness, regular sequence, Hilbert function, blowing up, associated graded ring. 
give some cases for the converse implications (Corollaries (1.13)-(1.15); see also the Remark following Corollary (2.5)). It turns out that some innocent looking conditions on the exceptional divisor (like depth $>0$, reduced, Cohen-Macaulay) have fairly strong implications for the center of blowing up.

In $\$ 2$, the various properties of projective normal flatness in connection with Hilbert functions are proved. For normal flatness, these properties were first given by Bennett [2] (improved later in [15,8 and 12]). We give a numerical characterization of projective normal flatness (Corollary (2.3)), which is used to prove the transitivity property for projective normal flatness (Corollary (2.5)). We point out here that it was only with the use of Hilbert functions that we were able to prove this result, contrary to the transitivity of normal flatness.

One reason for our interest in projective normal flatness was Nesselmann's paper [11], where he extends Singh's result [15] to centers $I$ such that $R / I^{n+1}$ is CohenMacaulay for large $n$; if $R / I$ is regular, then this condition is slightly stronger than projective normal flatness. Theorems (2.7) and (2.8) generalize the results of Singh [15], Nesselmann [11] and Orbanz [12] to centers $I$ along which $R$ is projectively normally flat.

Finally we note that in most cases where we prove flatness of $I^{n} / I^{n+1}$, we have to restrict to the case $R / I$ regular. For our purpose, the appropriate adaption to the nonregular case is the condition depth $I^{n} / I^{n+1}=\operatorname{dim} R / I$. This explains why many of the results are stated in terms of regular sequences on $I^{n} / I^{n+1}$.

\section{Projective normal flatness and the Hironaka-Grothendieck homomorphism.} Before turning to local rings we give some results on graded rings. For this, let $A=\bigoplus_{n \geqslant 0} A_{n}$ be a graded noetherian ring, generated over $A_{0}$ by $A_{1}$. Furthermore, we assume that $A_{0}$ is local with maximal ideal m.

Definition. $A$ is called projectively flat over $A_{0}$ if $\operatorname{Proj}(A) \rightarrow \operatorname{Spec}\left(A_{0}\right)$ is a flat morphism.

Clearly, if $A$ is flat over $A_{0}$, then it is projectively flat over $A_{0}$. Recall (e.g. [3]) that for any integer $d>0$, the graded algebra $A^{(d)}$ is defined by $A^{(d)}=\bigoplus_{n \geqslant 0} A_{n d}$, and then $\operatorname{Proj}(A)$ and $\operatorname{Proj}\left(A^{(d)}\right)$ are canonically isomorphic. If $M$ is a graded $A$-module and if $P$ is a homogeneous prime ideal of $A$, let $S$ be the set of homogeneous elements of $A \backslash P$. Then $M_{(P)}$ denotes the submodule of $S^{-1} M$ consisting of the fractions $m / s, m \in M, s \in S$, and $m$ and $s$ are homogeneous of the same degree. The following simple lemma is given for reference, and the proof will be omitted.

LeMma (1.1). Let $M$ be a finitely generated graded A-module. Then the following conditions are equivalent:

(1) $M_{n}=0$ for large $n$.

(2) $M_{(P)}=0$ for all $P \in \operatorname{Proj}(A)$.

Proposition (1.2). The following conditions are equivalent:

(1) $A$ is projectively flat over $A_{0}$.

(2) $A_{n}$ is flat over $A_{0}$ for $n$ large.

(3) There exists a $d$ such that $A^{(d)}$ is flat over $A_{0}$. 
Proof. (2) $\Rightarrow(3)$ is trivial, and $(3) \Rightarrow(1)$ since $\operatorname{Proj}(A)=\operatorname{Proj}\left(A^{(d)}\right)$. So it remains to prove $(1) \Rightarrow(2)$. For this we embed $A$ into an exact sequence

$$
0 \rightarrow I \rightarrow A_{0}[X] \rightarrow A \rightarrow 0,
$$

where $X=X_{1}, \ldots, X_{s}$ are finitely many indeterminates which are mapped to some system of generators of the $A_{0}$-module $A_{1}$. Let $k=A_{0} / \mathrm{m}$ and put $T=\operatorname{Tor}_{1}^{A_{0}}(A, k)$. Then $T=\bigoplus_{n \geqslant 0} T_{n}$, where $T_{n}=\operatorname{Tor}_{1}^{A_{0}}\left(A_{n}, k\right)$, and from the exact sequence (A) we conclude that

$$
T \simeq(I \cap \mathrm{m}[X]) /(I \mathrm{~m}[X]) .
$$

So we see that not only does $T$ have a natural structure of a graded $A$-module but, moreover, it is finitely generated over $A$. Now take any $P \in \operatorname{Proj}(A)$. Then

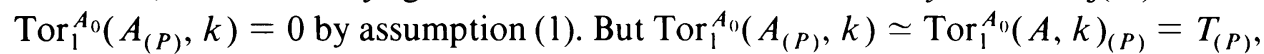
so by Lemma (1.1) we know that $T_{n}=0$ for large $n$, and this proves (2).

COROllary (1.3). The condition " $A_{n}$ is flat over $A_{0}$ for $n$ large" is open over $\operatorname{Spec}\left(A_{0}\right)$.

Proof. This follows from the equivalence of (1) and (2) above (see [9] for the corresponding discussion in the case of normal flatness).

Next we recall the definition of a weakly regular sequence in a graded ring, which was introduced in [5] (see also [6]).

Definition. Let $A=\bigoplus_{n \geqslant 0} A_{n}$ be a graded ring and let $\mathbf{x}=\left(x_{1}, \ldots, x_{r}\right)$ be a sequence of homogeneous elements of $A . \mathbf{x}$ is said to be weakly regular if there exists an integer $n_{0}$ with the property: For every $i \in\{1, \ldots, r\}$ and every $a \in A_{n}, a x_{i} \in$ $x_{1} A+\cdots+x_{i-1} A$ and $n \geqslant n_{0}$ imply $a \in x_{1} A+\cdots+x_{i-1} A$ (where $\left.x_{0}=0\right)$.

Given $A$ and $\mathbf{x}$ as above, recall that the Koszul complex $K .(\mathbf{x}, A)=\bigoplus_{i} K_{i}(\mathbf{x}, A)$ is defined by

$$
K_{i}(\mathbf{x}, A)=\stackrel{i}{\wedge}\left(\bigoplus_{j=1}^{r} A\left(-d_{j}\right)\right), \quad d_{j}=\operatorname{deg} x_{j} .
$$

Hence each $K_{i}(\mathbf{x}, A)$ has a natural structure as a graded $A$-module, and the same is true for the homology groups $H_{i}(\mathbf{x}, A)$. The homogeneous part of degree $n$ of $H_{i}(\mathbf{x}, A)$ will be denoted by $H_{i}(\mathbf{x}, A)_{n}$.

Now our assumptions made previously on $A$ are in force again, i.e. $A_{0}$ is local and $A$ noetherian, generated over $A_{0}$ by $A_{1}$. Furthermore, a sequence $\mathbf{x}=\left(x_{1}, \ldots, x_{r}\right)$ of homogeneous elements of $A$ will be fixed.

LEMMA (1.4). Consider the following conditions:

(1) $\mathbf{x}$ is a weakly regular sequence.

(2) $H_{1}(\mathbf{x}, A)_{n}=0$ for large $n$.

Then $(1) \Rightarrow(2)$, and if $d_{j}=\operatorname{deg} x_{j}=0$ for $j=1, \ldots, r$, then (1) and (2) are equivalent.

Proof. The proof of $(1) \Rightarrow(2)$ is literally the same as for regular sequences (see e.g. [14]). If $d_{j}=0$ for $j=1, \ldots, r$, then $H_{1}\left(\mathbf{x}, A_{n}\right)=H_{1}(\mathbf{x}, A)_{n}$. Therefore, $H_{1}(\mathbf{x}, A)_{n}$ $=0$ if and only if $\mathbf{x}$ is a regular sequence on $A_{n}$, which proves (2) $\Rightarrow(1)$. 
COROLlaRY (1.5). If $\mathbf{x}$ is a weakly regular sequence and if $\operatorname{deg} x_{j}=0$ for $j=1, \ldots, r$, then any permutation of $\mathbf{x}$ is a weakly regular sequence.

Proof. Condition (2) of (1.4) is independent of the order of $\mathbf{x}$.

Remark. Corollary (1.5), as well as Lemma (1.4), cannot be extended to a sequence $\mathbf{x}$ whose elements have positive degrees, as the following easy example shows. Take $A=k[[x]][Z] /\left(x Z^{2}\right)$, graded with respect to $Z$. Then the sequence $\left(Z^{2}, x Z\right)$ is weakly regular, but $x Z$ is not a weakly regular element.

Proposition (1.6). With $A$ and $\mathbf{x}$ as above, assume that $\operatorname{deg} x_{j}=0$ for $j=1, \ldots, r$. Then the following conditions are equivalent:

(1) $\mathbf{x}$ is a weakly regular sequence.

(2) $H_{1}(\mathbf{x}, A)_{n}=0$ for $n$ large.

(3) $H_{1}\left(\mathbf{x}, A_{(P)}\right)=0$ for all $P \in \operatorname{Proj}(A)$.

(4) $H_{1}\left(\mathbf{x}, A_{(P)}\right)=0$ for all $P \in \operatorname{Proj}(A)$ such that $\mathbf{x} A \subset P$.

Proof. The equivalence of (1) and (2) was shown in Lemma (1.4), while the equivalence of (3) and (4) follows from the well-known fact that $\mathbf{x} H_{1}\left(\mathbf{x}, A_{(P)}\right)=0$. Finally, (2) and (3) are equivalent by Lemma (1.1) since $H_{1}\left(\mathbf{x}, A_{(P)}\right)=H_{1}(\mathbf{x}, A)_{(P)}$. (Note that in (3) and (4) it is enough to check for those $P$ which are maximal in $\operatorname{Proj}(A)$.)

From now on let $R$ be a local ring with maximal ideal $\mathrm{m}$, and let $I$ be a proper ideal in $R$. We want to apply the results obtained so far to $A=G_{I}(R)$, the associated graded ring of $R$ with respect to $I$. Furthermore, we fix a sequence $x=\left(x_{1}, \ldots, x_{r}\right)$ of elements of $R$, we denote by $\mathbf{x} R$ the ideal generated by $\mathbf{x}$, and finally we put $I(\mathbf{x})=I+\mathbf{x} R$. We will use the notation $\mathrm{Bl}_{I}(R)$ for the class of homomorphisms $R \rightarrow R_{1}$ obtained by blowing up $R$ with center $I$. Sometimes $\mathbf{x}$ will be considered as a sequence in $G_{I}(R)$, by which we mean the sequence of the images of $x_{1}, \ldots, x_{r}$ in $R / I=G_{I}(R)_{0}$.

Using this notation we can state the following consequence of Proposition (1.6).

COROLlaRY (1.7). The following conditions are equivalent:

(1) $\mathbf{x}$ is weakly regular in $G_{I}(R)$.

(2) For any homomorphism $R \rightarrow R_{1}$ in $\mathrm{Bl}_{I}(R), \mathbf{x}$ is a regular sequence on $R_{1} / I R_{1}$.

Note again that in (2) above it is enough to consider those $R_{1}$ for which $R_{1} / I R_{1} \simeq\left[G_{I}(R)\right]_{(P)}$ with some maximal $P \in \operatorname{Proj}\left(G_{I}(R)\right)$.

DEFINITION. $R$ will be called projectively normally flat along $I$ if $G_{I}(R)$ is projectively flat over $G_{I}(R)_{0}=R / I$.

So by Proposition (1.2), $R$ is projectively normally flat along $I$ if and only if $I^{n} / I^{n+1}$ is flat over $R / I$ for large $n$.

LEMMA (1.8). Assume that $\mathbf{x}$ is a regular sequence on $R / I$. Then the following conditions are equivalent:

(1) $R$ is projectively normally flat along $I$.

(2) $G_{I}(R) \otimes_{R} R / I(\mathbf{x})$ is projectively flat over $R / I(\mathbf{x})$ and $\mathbf{x}$ is a weakly regular sequence in $G_{I}(R)$. 
Proof. (1) $\Rightarrow(2)$. The first part of (2) is obvious; as for the second part, note that $H_{1}(\mathbf{x}, R / I)=0$ implies $H_{1}\left(\mathbf{x}, I^{n} / I^{n+1}\right)=0$ as soon as $I^{n} / I^{n+1}$ is flat over $R / I$. Therefore, $\mathbf{x}$ is weakly regular in $G_{I}(R)$ by Proposition (1.2).

$(2) \Rightarrow(1)$. Since $\mathbf{x}$ is regular on $R / I, H_{1}\left(\mathbf{x}, I^{n} / I^{n+1}\right)=0$ implies $\operatorname{Tor}_{1}^{R / I}\left(R / I(\mathbf{x}), I^{n} / I^{n+1}\right)=0$ by [13, Lemma 1.4]. So we conclude by the local criterion of flatness [1, p. 91].

EXAMPLE. Let $R=k[[X, Y]] /\left(X Y, Y^{2}\right)=k[[x, y]], k$ any field, and take $I=y R$, $\mathbf{x}=(x)$. It follows that $G_{I}(R) \simeq k[[X]][T] /\left(X T, T^{2}\right)$. Then $\mathbf{x}$ is regular on $R / I$, but $x$ is a zero-divisor on $R=R / I^{n}$ for $n \geqslant 2$. On the other hand, $x$ is weakly regular in $G_{I}(R)$, since it is not a zero-divisor on $I^{n} / I^{n+1}=0$ for $n \geqslant 2$. This example shows that the property that $\mathbf{x}$ is weakly regular in $G_{I}(R)$ is different from the property that $\mathbf{x}$ is a regular sequence on $R / I^{n+1}$ for large $n$. The next result shows that this difference in the example is due to the fact that $x$ is a zero-divisor in $R$.

Proposition (1.9). The following conditions are equivalent:

(1) $\mathbf{x}$ is a regular sequence on $R / I^{n+1}$ for $n$ large.

(2) $\mathbf{x}$ is a regular sequence in $R$ and a weakly regular sequence in $G_{I}(R)$.

(3) $\mathbf{x}$ is a regular sequence in $R$ and $I^{n} \cap \mathbf{x} R=I^{n} \mathbf{x} R$ for $n$ large.

Furthermore, if these conditions are satisfied, then

$$
x_{i} \notin I+x_{1} R+\cdots+x_{i-1} R+x_{i+1} R+\cdots+x_{r} R
$$

for $1 \leqslant i \leqslant r$.

ProOF. (1) $\Rightarrow(2)$. From the exact sequences

$$
0 \rightarrow I^{n} / I^{n+1} \rightarrow R / I^{n+1} \rightarrow R / I^{n} \rightarrow 0
$$

and the induced sequences for the Koszul homology it is clear that (1) implies the second part of (2). To prove the first part, let $i \in\{1, \ldots, r\}$ and assume

$$
a x_{i} \in x_{1} R+\cdots+x_{i-1} R \quad \text { for some } a \in R \text {. }
$$

By (1) we conclude $a \in x_{1} R+\cdots+x_{i-1} R+I^{n}$ for $n$ large; hence, $a \in x_{1} R$ $+\cdots+x_{i-1} R$ by Krull's intersection theorem.

(2) $\Rightarrow(3)$. Choose integers $k$ and $c$ such that $\mathbf{x}$ is regular on $I^{n} / I^{n+1}$ for $n \geqslant k$ and such that

$$
I^{n+c} \cap \mathbf{x} R=I^{n}\left(I^{c} \cap \mathbf{x} R\right) \text { for all } n \geqslant 0
$$

(Artin-Rees-Lemma). To prove (3), it is obviously enough to show $I^{c+k} \cap \mathbf{x} R=$ $I^{c+k} \mathbf{x} R$, and since $I^{c+k} \cap \mathbf{x} R \subset I^{k} \mathbf{x} R \cap I^{c+k}$, actually it suffices to prove

$$
I^{n} \mathbf{x} R \cap I^{n+1} \subset I^{n+1} \mathbf{x} R \text { for } n \geqslant k .
$$

By the choice of $k$ and since $\mathbf{x}$ is a regular sequence in $R$, we have

$$
\operatorname{Tor}_{1}^{R}\left(R / \mathbf{x} R, I^{n} / I^{n+1}\right)=0 \text { for } n \geqslant k
$$

by [13, Lemma 1.4]. Therefore from (A) we deduce the exact sequences

$$
0 \rightarrow \operatorname{Tor}_{1}^{R}\left(R / \mathbf{x} R, R / I^{n+1}\right) \rightarrow \operatorname{Tor}_{1}^{R}\left(R / \mathbf{x} R, R / I^{n}\right), \quad n \geqslant k .
$$


This can also be written as

$$
0 \rightarrow\left(I^{n+1} \cap \mathbf{x} R\right) /\left(I^{n+1} \mathbf{x} R\right) \rightarrow\left(I^{n} \cap \mathbf{x} R\right) /\left(I^{n} \mathbf{x} R\right), \quad n \geqslant k,
$$

and therefore (B) above follows.

(3) $\Rightarrow(1)$. Since $\mathbf{x}$ is regular in $R, K .(\mathbf{x}, R)$ is a finite free resolution of $R / \mathbf{x} R$, and therefore

$$
H_{1}\left(\mathbf{x}, R / I^{n}\right) \simeq \operatorname{Tor}_{1}^{R}\left(R / \mathbf{x} R, R / I^{n}\right) \simeq\left(I^{n} \cap \mathbf{x} R\right) /\left(I^{n} \mathbf{x} R\right),
$$

proving (1).

To prove the last claim, observe that the conditions (1)-(3) are independent of the order of $\mathbf{x}$, so we may assume $i=r$. Now if $x_{r} \in I+x_{1} R+\cdots+x_{r-1} R$, then also $x_{r}^{n} \in I^{n}+x_{1} R+\cdots+x_{r-1} R$ for all $n$, contradicting the assumption that $\mathbf{x}$ is a regular sequence on $R / I^{n+1}$ for $n$ large.

REMARK. Let $R \rightarrow R_{1}$ be a homomorphism in $\mathrm{Bl}_{I}(R)$ and choose $t \in I$ such that $I R_{1}=t R_{1}$. Assume that $R / I^{n+1}$ is Cohen-Macaulay for large $n$. In [11, Lemma 3.1], the existence of elements $x_{1}, \ldots, x_{r}(r=\operatorname{dim} R / I)$ is shown with the property that $x_{1}, \ldots, x_{r}$ is a system of parameters with respect to $I$ and $t, x_{1}, \ldots, x_{r}$ is a regular sequence in $R_{1}$. Proposition (1.9) combined with Corollary (1.7) shows that this result of Nesselmann's is void in the sense that under the assumptions made above, any system $\mathbf{x}$ of parameters with respect to $I$ gives rise to a regular sequence $t$, $x_{1}, \ldots, x_{r}$ on $R_{1}$.

Following [13, Lemma 1.6], we recall that there are two canonical homomorphisms of graded rings

$$
g(I, \mathbf{x}): G_{I}(R) \otimes_{R} R / I(\mathbf{x}) \rightarrow G_{I(\mathbf{x}) / \mathbf{x} R}(R / \mathbf{x} R)
$$

and

$$
G(I, \mathbf{x}):\left(G_{I}(R) \otimes_{R} R / I(\mathbf{x})\right)\left[T_{1}, \ldots, T_{r}\right] \rightarrow G_{I(\mathbf{x})}(R) .
$$

(The second one is the Hironaka-Grothendieck homomorphism mentioned in the title of this section.)

THEOREM (1.10). With the notations introduced above, the following conditions are equivalent:

(1) $(\operatorname{Ker} g(I, \mathbf{x}))_{n}=0$ for large $n$, and $\mathbf{x}$ is a regular sequence in $R$.

(2) $\mathbf{x}$ is a weakly regular sequence in $G_{I}(R)$ and a regular sequence in $R$.

(3) $\mathbf{x}$ is a regular sequence on $R / I^{n+1}$ for large $n$.

Proof. For any $n$ we have $(\operatorname{Ker} g(I, \mathbf{x}))_{n}=0$ if and only if $I^{n} \cap \mathbf{x} R \subset I^{n} \mathbf{x} R+$ $I^{n+1}$. From this, one deduces easily that $(\operatorname{Ker} g(I, \mathbf{x}))_{n}=0$ for large $n$ if and only if $I^{n} \cap \mathbf{x} R=I^{n} \mathbf{x} R$ for large $n$ (compare [13, Lemma 1.7]). So the theorem follows from Proposition (1.9).

We note that Theorem (1.10) is parallel to [13, Theorem (1.8)], with the important difference that for large degrees there is no reasonable equivalent property involving $G(I, \mathbf{x})$. 
Combining the last result with Lemma (1.8) we obtain

Theorem (1.11). Assume, in addition, that $\mathbf{x}$ is a regular sequence on $R / I$. Then the following conditions are equivalent:

(1) $R$ is projectively normally flat along $I$ and $\mathbf{x}$ is a regular sequence in $R$.

(2) $G_{I}(R) \otimes_{R} R / I(\mathbf{x})$ is projectively flat over $R / I(\mathbf{x})$ and $\mathbf{x}$ is a weakly regular sequence in $G_{I}(R)$ as well as a regular sequence in $R$.

(3) $G_{I}(R) \otimes_{R} R / I(\mathbf{x})$ is projectively flat over $R / I(\mathbf{x})$ and $\mathbf{x}$ is a regular sequence on $R / I^{n+1}$ for large $n$.

(4) $(\operatorname{Ker} g(I, \mathbf{x}))_{n}=0$ for large $n, R / \mathbf{x} R$ is projectively normally flat along $I(\mathbf{x}) / \mathbf{x} R$, and $\mathbf{x}$ is a regular sequence in $R$.

Before turning to some consequences of Theorem (1.11), we make the following simple observation.

LEMMA (1.12). Let $A$ be a graded ring (of the type considered at the beginning) and let $K \subset A_{+}$be a homogeneous ideal. Consider the conditions:

(1) $K=0$.

(2) $K_{n}=0$ for large $n$.

(3) $K$ is nilpotent.

Then:

(a) (1) $\Rightarrow(2) \Rightarrow(3)$.

(b) If $A_{+}$contains a homogeneous nonzero-divisor, then (1) $\Leftrightarrow$ (2).

(c) If $\operatorname{Proj}(A)$ is reduced, then (2) $\Leftrightarrow(3)$.

Proof. (a) is obvious. If $a \in A_{+}$is a homogeneous nonzero-divisor and $k \in K_{s}$ is arbitrary, then (2) implies $a^{n} k=0$ for $n$ large, and therefore $k=0$, which proves (b). For (c) we note that if $K$ is nilpotent and $\operatorname{Proj}(A)$ is reduced, then $K_{(P)}=0$ for all $P \in \operatorname{Proj}(A)$, and the assertion follows from Lemma (1.1).

COROLlary (1.13). Let $P \subset R$ be a prime ideal for which $R / P$ is regular, and let $\mathbf{x}$ be a regular system of parameters with respect to $P$. Assume, furthermore, that:

(a) $\mathbf{x}$ is a regular sequence in $R$.

(b) $R$ is projectively normally flat along $P$.

(c) $\operatorname{depth}\left(G_{P}(R) \otimes_{R} R / \mathrm{m}\right)>0$.

Then $P$ is permissible in $R$.

Proof. By [13, Theorem 1.9], we have to show that $g(P, \mathbf{x})$ is an isomorphism. Applying Lemma (1.12)(b) to $K=\operatorname{Ker} g(P, \mathbf{x})$, we see that it suffices to prove $K_{n}=0$ for large $n$. But this follows from assumption (b) together with Theorem (1.11).

COROLlary (1.14). Let $R, P, \mathbf{x}$ be as in (1.13) and assume that:

(a) $R / P^{n}$ is Cohen-Macaulay for large $n$.

(b) $\operatorname{depth}\left(G_{P}(R) \otimes_{R} R / \mathrm{m}\right)>0$.

Then $P$ is permissible in $R$. 
Proof. By Lemma (1.8), Proposition (1.9) and assumption (a), $\mathbf{x}$ is a regular sequence in $R$, and $R$ is projectively normally flat along $P$. Therefore, the assertion follows from Corollary (1.13).

Corollary (1.15). Let $X$ be a locally noetherian Cohen-Macaulay scheme and let $Y$ be a regular closed subscheme. Denote by $\pi^{\prime}: X^{\prime} \rightarrow X$ the blowing up of $X$ along $Y$, and by $\pi: E \rightarrow Y$ the restriction of $\pi^{\prime}$ to the exceptional divisor. Let $y \in Y$. If $X$ is equimultiple along $Y$ at $y$ and if the fibre $E_{y}$ of $\pi$ is reduced, then $\pi$ is flat at $y$.

Proof. Let $R=\vartheta_{X, y}$, let $P$ be the ideal of $Y$ in $R$, and let $\mathbf{x}$ be a regular system of parameters with respect to $P$. Then from [10] (see also [6, Theorem 3]) we know that $g(P, \mathbf{x})$ has a nilpotent kernel. Since $E_{y}=\operatorname{Proj}\left(G_{P}(R)\right)$ is reduced, Lemma (1.12) tells us that $(\operatorname{Ker} g(P, \mathbf{x}))_{n}=0$ for large $n$, or, by Theorem $(1.11)$, that $R$ is projectively normally flat along $P$. This means by definition that $\pi$ is flat at $y$. (Note that $\mathbf{x}$ is a regular sequence in $R$; compare the Remark following Theorem (2.4).)

As an application of the techniques developed so far we prove the following transitivity property, which will be an essential step for the numerical characterization of projective normal flatness to be proved in Theorem (2.2).

TheOREM (1.16). Let $I_{1}=I+x_{1} R$ and $\mathbf{y}=\left(x_{2}, \ldots, x_{r}\right)($ so $r>0)$. If $\mathbf{x}$ is regular on $R / I^{n+1}$ for large $n$, then $\mathbf{y}$ is regular on $R / I_{1}^{n+1}$ for large $n$.

Proof. By Proposition (1.9) it is enough to show that $\mathbf{y}$ is weakly regular in $G_{I_{1}}(R)$ (note that $x_{i} \notin I_{1}$ for $i=2, \ldots, r$ by the last claim of Proposition (1.9)). So by Corollary (1.7) we have to show that for any homomorphism $R \rightarrow R_{1}$ in $\mathrm{Bl}_{I_{1}}(R), \mathrm{y}$ is a regular sequence on $R_{1} / I_{1} R_{1}$, and we may assume, in addition, that $R \rightarrow R_{1}$ is local. Now either $I_{1} R_{1}=x_{1} R_{1}$ or $I_{1} R_{1}=t R_{1}$ for some $t \in I$. These two cases will be treated separately.

Case 1. $I_{1} R_{1}=x_{1} R_{1}$. Let $i \in\{1, \ldots, r\}$ and assume

$$
z x_{i}=a_{1} x_{1}+\cdots+a_{i-1} x_{i-1}, \quad z, a_{1}, \ldots, a_{i-1} \in R\left[I x_{1}^{-1}\right] .
$$

Now from Proposition (1.9) we know

$$
I^{n} \cap\left(x_{1} R+\cdots+x_{i-1} R\right)=I^{n}\left(x_{1} R+\cdots+x_{i-1} R\right), \quad n \text { large. }
$$

Write

$$
z=y / x_{1}^{n}, \quad a_{j}=b_{j} / x_{1}^{n}, \quad j=1, \ldots, i-1, y, b_{1}, \ldots, b_{i-1} \in I^{n},
$$

where $n$ is chosen big enough to satisfy (B). Then (A) implies $y x_{i}=b_{1} x_{1}$ $+\cdots+b_{i-1} x_{i-1}$, and since $\mathbf{x}$ is a regular sequence in $R$, we obtain

$$
y \in I^{n} \cap\left(x_{1} R+\cdots+x_{i-1} R\right)=I^{n}\left(x_{1} R+\cdots+x_{i-1} R\right) .
$$

so we may write

$$
y=c_{1} x_{1}+\cdots+c_{i-1} x_{i-1}, \quad c_{1}, \ldots, c_{i-1} \in I^{n} .
$$

Therefore

$$
z=y / x_{1}^{n}=\left(c_{1} / x_{1}^{n}\right) x_{1}+\cdots+\left(c_{i-1} / x_{1}^{n}\right) x_{i-1} .
$$

This proves that $x_{1}, \ldots, x_{r}$ is a regular sequence in $R\left[I x_{1}^{-1}\right]$ and therefore also in its localization $R_{1}$. 
Case 2. $I_{1} R_{1}=t R_{1}, t \in I$. Then $R_{1}$ is a localization of $R\left[I_{1} t^{-1}\right]=R\left[I t^{-1}\right]\left[x_{1} t^{-1}\right]$. Let $\mathrm{m}_{1}$ denote the maximal ideal of $R_{1}$ and let $S=R\left[I t^{-1}\right]_{\mathrm{m}_{1} \cap R\left[I t^{-1}\right]}$. Then $R \rightarrow S$ is in $\mathrm{Bl}_{I}(R)$ and $R_{1}$ is obtained from $S$ by blowing up the ideal $t S+x_{1} S$, which, by Corollary (1.7), is generated by a regular sequence. Therefore, the homomorphism

$$
S /\left(t S+x_{1} S\right) \rightarrow R_{1} /\left(t R_{1}+x_{1} R_{1}\right)=R_{1} / t R_{1}=R_{1} / I_{1} R_{1}
$$

is flat. Since $\mathbf{x}$ is regular on $S / t S$, the flatness above implies that $\mathbf{y}$ is regular on $R_{1} / I_{1} R_{1}$.

REMARK. The theorem above remains true if "large n" is replaced by "all $n$ ". In fact, in this case the proof can be obtained much easier by using the techniques of [13].

2. Numerical properties of projective normal flatness. We keep the notation of $\S 1$, so $R$ will denote a local ring with maximal ideal $\mathrm{m}$, and $I$ will be a proper ideal of $R$. Furthermore, $\mathbf{x}=\left(x_{1}, \ldots, x_{r}\right)$ is a sequence of elements of $R$, and from now on we assume that $\mathbf{x}$ is a system of parameters with respect to $I$, i.e. $r=\operatorname{dim} R / I$ and $I(\mathbf{x})$ is m-primary.

We recall from [8] that a generalized Hilbert function is defined by

$$
H^{(0)}[\mathbf{x}, I, R](n)=e\left(\mathbf{x}, I^{n} / I^{n+1}\right),
$$

where $e(\mathbf{x},-)$ denotes the multiplicity symbol introduced by Wright. We are going to use the fact that $e(\mathbf{x},-)$ is additive on exact sequences. For the properties of $e(\mathbf{x},-)$, as well as of the generalized Hilbert functions, the reader is referred to [8]. As usual we define

$$
H^{(i)}[\mathbf{x}, I, R](n)=\sum_{j=0}^{n} H^{(i-1)}[\mathbf{x}, I, R](j) \quad \text { if } i>0 .
$$

For $\mathbf{x}=\varnothing$, which means $r=0$, the functions $H^{(i)}[\mathbf{x}, I, R]$ are the well-known Hilbert functions of the m-primary ideal $I$ and will be denoted by $H^{(i)}[I, R]$. Finally we put $H^{(i)}[R]=H^{(i)}[\mathrm{m}, R]$.

Our next aim is to give a numerical criterion for $R$ to be projectively normally flat along $I$. We start with a technical result which will be needed in the inductive proof of the criterion.

Lemma (2.1). Assume $r>0$ and put $I_{1}=I+x_{1} R, \mathbf{y}=\left(x_{2}, \ldots, x_{r}\right)$. Let $n \geqslant 0$ be $a$ fixed integer and assume

$$
\operatorname{depth} I_{1}^{n} / I_{1}^{n+1}=r-1 \text { and } H^{(0)}\left[\mathbf{y}, I_{1}, R\right](n)=H^{(1)}[\mathbf{x}, I, R](n) \text {. }
$$

Then $x_{1}$ is not a zero-divisor on $I_{1}^{n} / I^{n+1}$.

Proof. Consider the exact sequence

$$
0 \rightarrow I_{1}^{n} / I^{n+1} \rightarrow R / I^{n+1} \rightarrow R / I_{1}^{n} \rightarrow 0 .
$$

Since $\operatorname{dim} R / I_{1}^{n}<r$, we obtain

$$
e\left(\mathbf{x}, I_{1}^{n} / I^{n+1}\right)=e\left(\mathbf{x}, R / I^{n+1}\right)=e\left(\mathbf{y}, I_{1}^{n} / I_{1}^{n+1}\right),
$$


the second equality by assumption. Now by definition

$$
\begin{aligned}
e\left(\mathbf{x}, I_{1}^{n} / I^{n+1}\right) & =e\left(\mathbf{y}, I_{1}^{n} / I^{n+1}+x_{1} I_{1}^{n}\right)-e\left(\mathbf{y}, \operatorname{Ann}\left(x_{1}, I_{1}^{n} / I^{n+1}\right)\right) \\
& =e\left(\mathbf{y}, I_{1}^{n} / I_{1}^{n+1}\right)-e\left(\mathbf{y}, \operatorname{Ann}\left(x_{1}, I_{1}^{n} / I^{n+1}\right)\right) .
\end{aligned}
$$

Comparing (A) and (B) yields

$$
e\left(\mathbf{y}, \operatorname{Ann}\left(x_{1}, I_{1}^{n} / I^{n+1}\right)\right)=0 .
$$

If $r=1$, then $(\mathrm{C})$ says that $\mathrm{l}\left(\operatorname{Ann}\left(x_{1}, I_{1}^{n} / I^{n+1}\right)\right)=0$ and therefore $x_{1}$ is regular on $I_{1}^{n} / I^{n+1}$. So assume now that $r>1$. For the rest of the proof we put $N_{j}=$ $\operatorname{Ann}\left(x_{1}^{j}, I_{1}^{n} / I^{n+1}\right), j=1,2, \ldots$ Then $0=N_{0} \subset N_{1} \subset \cdots \subset I_{1}^{n} / I^{n+1}$, and therefore there is a smallest integer $k \geqslant 0$ such that $N_{k}=N_{k+m}$ for all $m \geqslant 0$. The proof will be finished if we can show that $k=0$. By $(\mathrm{C})$ and the properties of the multiplicity symbol, we get

$$
\operatorname{dim} N_{1}<r-1
$$

Observe that $I^{n+1} N_{1}=0$ and $x_{1} N_{1}=0$, so $I_{1}^{n+1} N_{1}=0$. Therefore (D) implies that there exists some $y \in R$ such that

$$
y N_{1}=0, \quad y \notin P \quad \text { for all } P \in \operatorname{Assh}\left(R / I_{1}^{n+1}\right)=\operatorname{Assh}\left(R / I_{1}\right) .
$$

(Here we use the notation $\operatorname{Assh}\left(R / I_{1}\right)=\left\{P^{\prime} \in \operatorname{Ass}\left(R / I_{1}\right) \mid \operatorname{dim} R / P^{\prime}=\right.$ $\left.\operatorname{dim} R / I_{1}\right\}$.) Next we show, by induction on $j$, that

$$
y^{j} N_{j}=0 \text {. }
$$

This is clear for $j=1$ by (E). For $j>1$, let $z \in N_{j}$, so that by definition $x_{1}^{j} z=0=$ $x_{1}^{j-1}\left(x_{1} z\right)$. Then $x_{1} z \in N_{j-1}$, which implies $y^{j-1} x_{1} z=0$ by inductive assumption. But then $y^{j-1} z \in N_{1}$ gives $y\left(y^{j-1} z\right)=0$ by (E) again, so (F) is proved. The next step is to show that

$$
y \text { is regular on } I_{1}^{n} / I_{1}^{n+1} \text {. }
$$

Recall that $I_{1}^{n} / I_{1}^{n+1}$ is a Cohen-Macaulay module of dimension $r-1$ by assumption. Therefore $(\mathrm{G})$ follows from $(\mathrm{E})$. After these preparations assume now, if possible, that $k>0$ and choose $z \in N_{k} \backslash N_{k-1}$. Then $y^{k} z=0$, so (G) implies

$$
z \in I_{1}^{n+1} / I^{n+1}=\left(I^{n+1}+x_{1} I_{1}^{n}\right) / I^{n+1},
$$

and therefore $z=x_{1} z_{1}$ for some $z_{1} \in I_{1}^{n} / I^{n+1}$. Finally, $z \in N_{k}$ implies $z_{1} \in N_{k+1}=$ $N_{k}$, which leads to the contradiction $x_{1}^{k-1} z=0$.

To state the criterion announced before, we need some more notation. For $i \in\{0, \ldots, r-1\}$ we put

$$
I_{i}=I+x_{1} R+\cdots+x_{i} R\left(\text { so } I_{0}=I\right) \text { and } \mathbf{y}_{i}=\left(x_{i+1}, \ldots, x_{r}\right) .
$$

THEOREM (2.2). With the notations introduced above, consider the conditions:

(1) $R / I^{n+1}$ is Cohen-Macaulay for large $n$.

(2) For all $i \in\{0, \ldots, r-1\}$ we have $H^{(0)}\left[\mathbf{y}_{i+1}, I_{i+1}, R\right](n)=H^{(1)}\left[\mathbf{y}_{i}, I_{i}, R\right](n)$ for large $n$.

(3) depth $I^{n} / I^{n+1}=r$ for large $n$.

Then $(1) \Rightarrow(2) \Rightarrow(3)$, and if $\mathbf{x}$ is a regular sequence in $R$, then all three conditions are equivalent. 
Proof. (1) $\Rightarrow$ (2). We make induction on $r$. Condition (2)is void in the case $r=0$, so assume $r>0$. From Theorem (1.16) we know that $R / I_{1}^{n+1}$ is Cohen-Macaulay for $n$ large, and therefore condition (2) is satisfied for $1 \leqslant i \leqslant r-1$ by inductive assumption. Furthermore, for $n$ large, $x_{1}$ is regular on the submodule $I_{1}^{n} / I^{n+1}$ of $R / I^{n+1}$, which implies

$$
\begin{aligned}
e\left(\mathbf{x}, I_{1}^{n} / I^{n+1}\right) & =e\left(\mathbf{y}_{1}, I_{1}^{n} / I^{n+1}+x_{1} I_{1}^{n}\right)=e\left(\mathbf{y}_{1}, I_{1}^{n} / I_{1}^{n+1}\right) \\
& =H^{(0)}\left[\mathbf{y}_{1}, I_{1}, R\right](n) .
\end{aligned}
$$

On the other hand, as in the proof of (2.1) we have

$$
e\left(\mathbf{x}, I_{1}^{n} / I^{n+1}\right)=e\left(\mathbf{x}, R / I^{n+1}\right)=H^{(1)}[\mathbf{x}, I, R](n),
$$

which proves (2) in the remaining case $i=0$.

(2) $\Rightarrow(3)$. We use induction on $r$ again, the case $r=0$ being trivial. For $r>0$ the inductive assumption implies depth $I_{1}^{n} / I_{1}^{n+1}=r-1$ for large $n$, which means that $x_{2}, \ldots, x_{r}$ is a regular sequence on $I_{1}^{n} / I_{1}^{n+1}$ for these $n$. By Lemma (2.1), $x_{1}$ is regular on $I_{1}^{n} / I^{n+1}$ for $n$ large, and since $\left(I_{1}^{n} / I^{n+1}\right) / x_{1}\left(I_{1}^{n} / I^{n+1}\right) \simeq I_{1}^{n} / I_{1}^{n+1}$, we conclude that $\mathbf{x}$ is a regular sequence on $I_{1}^{n} / I^{n+1}$ for large $n$. Now consider the exact sequences

$$
\begin{gathered}
0 \rightarrow I_{1}^{n+1} / I^{n+1} \rightarrow I_{1}^{n} / I^{n+1} \rightarrow I_{1}^{n} / I_{1}^{n+1} \rightarrow 0, \\
0 \rightarrow I^{n+1} / I^{n+2} \rightarrow I_{1}^{n+1} / I^{n+2} \rightarrow I_{1}^{n+1} / I^{n+1} \rightarrow 0 .
\end{gathered}
$$

Since for large $n$

$$
\operatorname{depth} I_{1}^{n} / I_{1}^{n+1}=r-1 \text { and } \operatorname{depth} I_{1}^{n} / I^{n+1}=r \text {, }
$$

(S1) implies depth $I_{1}^{n+1} / I^{n+1}=r$ for large $n$. Using this and depth $I_{1}^{n+1} / I^{n+2}=r$ again, (3) follows from (S2).

(3) $\Rightarrow(1)$. This was shown in Proposition (1.9).

REMARK. Conditions (1) and (2) in the theorem above are independent, not only of the order of the sequence $\mathbf{x}$, but also of the choice of the $x$ 's. So the same is true for condition (2) as long as $\mathbf{x}$ is a regular sequence in $R$.

In the special case that $R / I$ is regular, the condition depth $I^{n} / I^{n+1}=r$ is equivalent to the flatness of $I^{n} / I^{n+1}$ over $R / I$. Therefore, we get the following

COROLlary (2.3). Assume that $R / I$ is regular and that $\mathbf{x}$ is a regular sequence in $R$. Then the following conditions are equivalent:

(1) $R$ is projectively normally flat along $I$.

(2) For all $i \in\{0, \ldots, r-1\}$ we have $H^{(0)}\left[\mathbf{y}_{i+1}, I_{i+1}, R\right](n)=H^{(1)}\left[\mathbf{y}_{i}, I_{i}, R\right](n)$ for large $n$.

The criteria (2.2) and (2.3) allow us to prove a transitivity property analogous to the transitivity of normal flatness.

THEOREM (2.4). Let $s \in\{1, \ldots, r\}$ be given and put $\mathbf{y}=\left(x_{1}, \ldots, x_{s}\right), \mathbf{z}=$ $\left(x_{s+1}, \ldots, x_{r}\right)$. Assume that $\mathbf{x}$ is a regular sequence in $R$. Then the following conditions are equivalent:

(1) $R / I^{n+1}$ is Cohen-Macaulay for large $n$. 
(2) (a) $R / I(\mathbf{y})^{n+1}$ is Cohen-Macaulay for large $n$ and (b) for all $P \in \operatorname{Assh}(R / I(\mathbf{y}))$, $R_{p} / I^{n+1} R_{p}$ is Cohen-Macaulay for large $n$.

Proof. (1) $\Rightarrow(2)$ follows from Theorem (1.16) by induction. For the converse, define $\tilde{\mathbf{y}}_{i}=\left(x_{i+1}, \ldots, x_{s}\right), 0 \leqslant i<s$. Then by (a) and Theorem (2.2), we have

$$
H^{(0)}\left[\mathbf{y}_{i+1}, I_{i+1}, R\right](n)=H^{(1)}\left[\mathbf{y}_{i}, I_{i}, R\right](n), \quad n \text { large, } s \leqslant i<r,
$$

and by (b) we know, for all $P \in \operatorname{Assh}(R / I(\mathbf{y}))$, that

$$
H^{(0)}\left[\tilde{\mathbf{y}}_{i+1}, I_{i+1} R_{p}, R_{p}\right](n)=H^{(1)}\left[\tilde{\mathbf{y}}_{i}, I_{i} R_{p}, R_{p}\right](n), \quad n \text { large, } 0 \leqslant i<s .
$$

Now the associativity law for multiplicities (see $[8$, p. 102)] gives (C)

$$
H^{(0)}\left[\mathbf{y}_{i+1}, I_{i+1}, R\right]=\sum_{P \in \operatorname{Assh}(R / I(\mathbf{y}))} e(\mathbf{z}, R / P) H^{(0)}\left[\tilde{\mathbf{y}}_{i+1}, I_{i+1} R_{p}, R_{p}\right], \quad i<s,
$$

and, similarly,

$$
H^{(1)}\left[\mathbf{y}_{i}, I_{i}, R\right]=\sum_{P \in \operatorname{Assh}(R / I(\mathbf{y}))} e(\mathbf{z}, R / P) H^{(1)}\left[\tilde{\mathbf{y}}_{i}, I_{i} R_{p}, R_{p}\right], \quad i<s .
$$

Substituting (B) into (C) and (D) shows that actually (A) holds for all $i \in\{0, \ldots, r$ -1 \}, which completes the proof in view of Theorem (2.2).

REMARK. If $R$ is projectively normally flat along $I$, then $\operatorname{ht}(I)=1(I)$, the analytic spread of $I$ [7, Theorem 1]. Conversely, assume that $\mathrm{ht}(I)=1(I)$. Then $\operatorname{dim} R=$ $\operatorname{dim} R / I+\operatorname{ht}(I)$ (see [7]) and, furthermore, there are elements $z_{1}, \ldots, z_{s}, s=\mathrm{ht}(I)$, which generate $I$ up to radical. Since $\operatorname{dim} R=r+s$ and since $x_{1}, \ldots, x_{r}, z_{1}, \ldots, z_{s}$ generate an m-primary ideal, we conclude that $x_{1}, \ldots, x_{r}$ are part of a system of parameters of $R$. So if $R$ is assumed to be Cohen-Macaulay, then $\mathbf{x}$ is necessarily a regular sequence in $R$.

Corollary (2.5). Let $P \subset Q$ be prime ideals of $R$ such that both $R / P$ and $R / Q$ are regular. Assume that $R$ is Cohen-Macaulay. Then the following conditions are equivalent:

(1) $R$ is projectively normally flat along $P$.

(2) $R$ is projectively normally flat along $Q$ and $R_{Q}$ is projectively normally flat along $P R_{Q}$

Proof. Let $\mathbf{x}$ be a regular system of parameters with respect to $P$ such that part of it generates $Q$ over $P$. We will show that both conditions (1) and (2) imply that $\mathbf{x}$ is a regular sequence in $R$, and then the result will follow from Theorem (2.4). Since $R$ is assumed to be Cohen-Macaulay, by the Remark above it is enough to show that $\mathrm{ht}(P)=1(P)$. Under assumption (1) this was proved in [7, Theorem 1]. So assume that (2) holds. Then by [7, Theorems 0 and 1] we see that $e(R)=e\left(R_{Q}\right)$ and $e\left(R_{Q}\right)=e\left(\left(R_{Q}\right)_{P R_{Q}}\right)$, which means that $e(R)=e\left(R_{p}\right)$. But this is equivalent to $\operatorname{ht}(P)=1(P)[4$, Satz 2; or 7, Theorem 0$]$.

EXAMPLE. A typical non-Cohen-Macaulay ring is

$$
R=k[[X, Y, Z]] /\left(X Z, Z^{2}\right)=k[[x, y, z]] .
$$


Let $P=z R$ and $Q=P+y R$. Then

$$
G_{P}(R) \simeq k[[X, Y]][T] /\left(X T, T^{2}\right)
$$

and hence $R$ is projectively normally flat along $P$. On the other hand,

$$
G_{Q}(R) \simeq k[[X]]\left[T_{1}, T_{2}\right] /\left(X T_{2}, T_{2}^{2}\right),
$$

which has torsion in every degree. So $R$ is not projectively normally flat along $Q$. This example shows that Corollary (2.5) does not remain true without any additional assumption on $R$.

REMARK. In [5] the following situation is studied: $P$ is a prime ideal in $R$ such that $R / P$ is regular. $R$ itself can be written as $S /\left(f_{1}, \ldots, f_{m}\right)$, where $(S, \mathfrak{M})$ is a regular local ring, and where $f_{1}, \ldots, f_{m}$ satisfy the following condition: If $P_{0}$ denotes the inverse image of $P$ in $S$, then the initial forms of $f_{1}, \ldots, f_{m}$ with respect to $P_{0}$ as well as to $\mathfrak{M}$ are a weakly regular sequence in the corresponding graded ring. Some effort is made in [5] to show that under these conditions $e(R)=e\left(R_{p}\right)$ implies a property of Hilbert functions which is weaker than (2) in Corollary (2.3). Our new method gives a better result as follows. Let $\pi: \operatorname{Proj}\left(G_{p}(R)\right) \rightarrow \operatorname{Spec}(R / P)$ be the natural morphism. By [4, Satz 2], $e(R)=e\left(R_{p}\right)$ implies that all fibres of $\pi$ have the same dimension. But since $R / P$ is regular and $\operatorname{Proj}\left(G_{p}(R)\right)$ is Cohen-Macaulay [5, Satz 2.1], $\pi$ must be flat (see e.g. [1, Proposition (3.5), p. 94]). So $R$ is projectively normally flat along $P$.

In the final part of the paper we study the behaviour of Hilbert functions under blowing up $R$ with center $I$, provided that $R$ is projectively normally flat along $I$. Recall that $\mathrm{Bl}_{I}(R)$ denotes the class of homomorphisms $R \rightarrow R_{1}$ of local rings obtained by blowing up $R$ with center $I$. If $I R_{1} \neq R_{1}$, then there is a unique homogeneous prime ideal $Q$ in $G_{I}(R)$ such that $R_{1} / I R_{1} \simeq\left[G_{I}(R)\right]_{(Q)}$. From now on we will only consider the case that $Q \cap G_{I}(R)_{0}=\mathrm{m} / I$, i.e. the homomorphism $R \rightarrow R_{1}$ is local. We put $s\left(R_{1}\right)=\operatorname{dim} G_{I}(R) / Q-1$. Then $s\left(R_{1}\right)$ is the dimension of the projective variety with homogeneous coordinate ring $G_{I}(R) / Q$, which is the same as the transcendence degree of the function field of this variety over $k=R / \mathrm{m}$. Since this function field is $k$-isomorphic to the residue field of $R_{1}$, we see that $s\left(R_{1}\right)$ is the residual transcendence degree of $R_{1}$ over $R$.

THEOREM (2.6). Let $R \rightarrow R_{1}$ be a local homomorphism in $\mathrm{Bl}_{I}(R)$ and let $s=s\left(R_{1}\right)$. Assume that $r=0$, i.e. I is m-primary. Then:

(a) $H^{(s+2)}\left[R_{1} / I R_{1}\right] \leqslant H^{(1)}[I, R]$ and

(b) $H^{(s+1)}\left[R_{1} / I R_{1}\right] \leqslant H^{(0)}[R]$ if $I=\mathrm{m}$.

Proof. This is a special case of the results in [12].

THEOREM (2.7). Let $R \rightarrow R_{1}$ be a local homomorphism in $\mathrm{Bl}_{I}(R)$ and let $s=s\left(R_{1}\right)$. Assume that depth $I^{n} / I^{n+1}=r$ for large $n$. (Recall that $\left.r=\operatorname{dim} R / I\right)$. Then:

(a) $H^{(s+2)}\left[R_{1} / I R_{1}\right] \leqslant H^{(1)}[I(\mathbf{x}), R]$ and

(b) $H^{(s+1)}\left[R_{1} / I R_{1}\right] \leqslant H^{(0)}[R]$ if $I(\mathbf{x})=\mathrm{m}$.

In particular, we have:

(a') $H^{(s+1)}\left[R_{1}\right] \leqslant H^{(1)}[I(\mathbf{x}), R]$ and

(b') $H^{(s)}\left[R_{1}\right] \leqslant H^{(0)}[R]$ if $I(\mathbf{x})=\mathrm{m}$. 
Proof. Let us note first that $I R_{1}=t R_{1}$ for some $t \in I$, and therefore, by [15, Theorem 1],

$$
H^{(i)}\left[R_{1}\right] \leqslant H^{(i+1)}\left[R_{1} / I R_{1}\right] \text { for all } i \geqslant 0 \text {. }
$$

So $\left(a^{\prime}\right)$ and $\left(b^{\prime}\right)$ follow from (a) and (b), respectively. To prove (a) and (b), we reduce to Theorem (2.6) by showing that the "origin" of a cylinder over $R_{1}$ can be viewed as a point obtained by blowing up $R$ with center $I(\mathbf{x})$.

By Corollary (1.7) and the assumption depth $I^{n} / I^{n+1}=r$ for large $n$ we know that $x_{1}, \ldots, x_{r}$ is a regular sequence on $R_{1} / I R_{1}=R_{1} / t R_{1}$. Let $I_{1}=t R_{1}+x_{1} R_{1}$ $+\cdots+x_{r} R_{1}$. Then $R_{1}\left[x_{1} / t, \ldots, x_{r} / t\right] / I_{1} R_{1}\left[x_{1} / t, \ldots, x_{r} / t\right]$ is isomorphic to the polynomial ring $\left(R_{1} / I_{1}\right)\left[X_{1}, \ldots, X_{r}\right], X_{i}$ corresponding to $x_{i} / t$. In this polynomial ring, let $\Re$ be the maximal ideal generated by the maximal ideal of $R_{1} / I_{1}$ and $X_{1}, \ldots, X_{r}$. Then, up to $R_{1}$-isomorphism, there is a unique local ring $R_{2}$ obtained by blowing up $R_{1}$ with center $I_{1}$ and such that

$$
R_{2} / I_{1} R_{2} \simeq\left(R_{1} / I_{1}\right)\left[X_{1}, \ldots, X_{r}\right]_{\mathfrak{R}} .
$$

So obviously, $H^{(0)}\left[R_{2} / I_{1} R_{2}\right]=H^{(r)}\left[R_{1} / I_{1}\right]$. On the other hand, by [15, Theorem 1] we have $H^{(0)}\left[R_{1} / I R_{1}\right]=H^{(0)}\left[R_{1} / t R_{1}\right] \leqslant H^{(r)}\left[R_{1} / I_{1}\right]$, which gives

$$
H^{(i)}\left[R_{1} / I R_{1}\right] \leqslant H^{(i)}\left[R_{2} / I_{1} R_{2}\right] \text { for all } i \geqslant 0 \text {. }
$$

Since $R\left[I(\mathbf{x}) t^{-1}\right]=R\left[I t^{-1}\right]\left[x_{1} / t, \ldots, x_{r} / t\right], R_{2}$ is obtained by blowing up $R$ with center $I(\mathbf{x})$ as well, and $I(\mathbf{x}) R_{2}=t R_{2}=I_{1} R_{2}$. Now the result follows from Theorem (2.6) and the fact that $s\left(R_{2}\right)=s$ since $R_{1}$ and $R_{2}$ have the same residue field.

REMARK. Theorem (2.7) is stronger than the result of Nesselmann [11] in various respects. Besides the fact that our method gives an estimate for the exceptional divisor instead of the blowing up itself, we note that our assumption on depth $I^{n} / I^{n+1}$ is weaker than that of Nesselmann (as was shown in an example in $\S 1$ ), and we could dispose of the assumption of excellence for $R$.

For convenience we restate the result in the classical case of a smooth center in geometric form.

THEOREM (2.8). Let $X$ be a locally noetherian scheme and let $Y$ be a regular closed subscheme. Let $\pi^{\prime}: X^{\prime} \rightarrow X$ be the blowing up of $X$ along $Y$ and denote by $\pi: E \rightarrow Y$ the restriction of $\pi^{\prime}$ to the exceptional divisor. Let $y \in Y$ and $x^{\prime} \in \pi^{-1}(y)$. If $\pi$ is flat at every point of $\pi^{-1}(y)$, then

$$
H^{(s)}\left[\Theta_{X^{\prime}, x^{\prime}}\right] \leqslant H^{(0)}\left[\Theta_{X, y}\right],
$$

where $s$ is the transcendence degree of $k\left(x^{\prime}\right)$ over $k(y)$.

\section{REFERENCES}

1. A. Altman and S. Kleiman, Introduction to Grothendieck duality theory, Lecture Notes in Math., vol. 146, Springer-Verlag, Berlin, Heidelberg and New York, 1970.

2. B. M. Bennett, On the characteristic functions of a local ring, Ann. of Math. (2) 91 (1970), 25-87.

3. R. Hartshorne, Algebraic geometry, Springer-Verlag, Berlin, Heidelberg and New York, 1977.

4. M. Herrmann and U. Orbanz, Faserdimensionen von A ufblasungen lokaler Ringe und Aquimultiplizität, J. Math. Kyoto.Univ. 20 (1980), 651-659.

5. 437-451 , Normale Flachheit und Äquimultiplizität für vollständige Durchschnitte, J. Algebra 70 (1981), 
6. B__ Between equimultiplicity and normal flatness, Algebraic Geometry, Proceedings La Rabida 1981 (ed. Aroca-Buchweitz-Giusti-Merle), Lecture Notes in Mathematics, vol. 961, Springer, Berlin and New York, 1982, pp. 200-232.

7. __ On equimultiplicity, Math. Proc. Cambridge Philos. Soc. 91 (1982), 207-213.

8. M. Herrmann, R. Schmidt and W. Vogel, Theorie der normalen Flachheit, Teubner Verlag, Leipzig, 1977.

9. H. Hironaka, Resolution of singularities of an algebraic variety over a field of characteristic zero. I, II, Ann. of Math. (2) 79 (1964), 109-326.

10. J. Lipman, Equimultiplicity, reduction and blowing up, Commutative Algebra-Analytic Methods (ed. R. Draper), Lecture Notes in Pure and Appl. Math., vol. 68, Dekker, New York and Basel, 1981.

11. D. Nesselmann, Über monoidale Transformations lokaler Ringe, Math. Nachr. 88 (1979), 285-294.

12. U. Orbanz, Multiplicities and Hilbert functions under blowing up, Manuscripta Math. 36 (1981), 179-186.

13. L. Robbiano, On normal flatness and some related topics, Commutative Algebra, Proceedings of the Trento Conference (ed. Greco-Valla), Lecture Notes in Pure and Appl. Math., vol. 84, Marcel Dekker, New York and Basel, 1983, pp. 235-251.

14. J.-P. Serre, Algèbre locale-Multiplicités, Lecture Notes in Math., vol. 11, Springer-Verlag, Berlin, Heidelberg and New York, 1965.

15. B. Singh, Effect of a permissible blowing up on the local Hilbert functions, Invent. Math. 26 (1974), $201-212$.

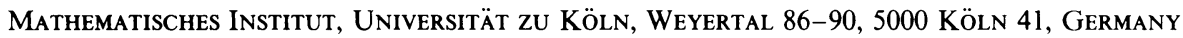

Istituto di Matematica, Università di Genova, Via L. B. Alberti 4, 16132 Genova, Italy 